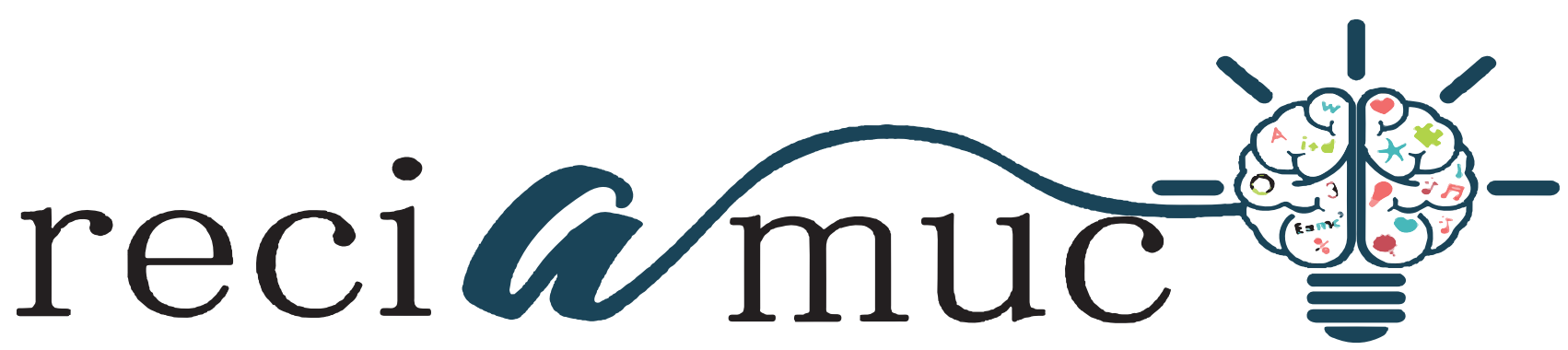

DOI: 10.26820/reciamuc/4.(1).enero.2020.114-126

URL: https://reciamuc.com/index.php/RECIAMUC/article/view/446

EDITORIAL: Saberes del Conocimiento

REVISTA: RECIAMUC

ISSN: $2588-0748$

TIPO DE INVESTIGACIÓN: Artículo de Revisión

CÓDIGO UNESCO: 3205 Medicina Interna

PAGINAS: $114-126$

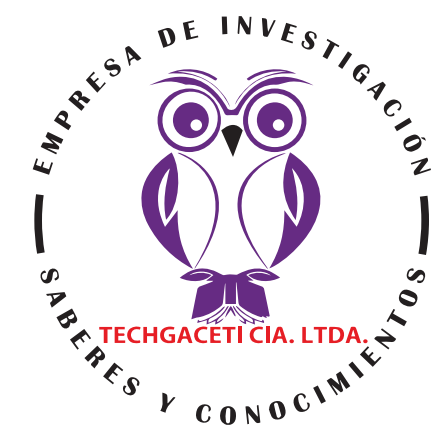

\title{
Insuficiencia renal crónica en pacientes con diabetes mellitus
}

\section{Chronic renal failure in patients with diabetes mellitus \\ Insuficiência renal crônica em pacientes com diabetes mellitus}

\section{Melissa Monserrat Carvajal Prado'; Kerly Paola Motoche Granados²; Maria Alicia Vera Carrera3; Shirley Mercedes Vite Correa ${ }^{4}$}

\section{RECIBIDO: 18/11/2019 ACEPTADO: 20/12/2019 PUBLICADO: 31/01/2020}

1. Médico; Investigadora Independiente; Guayaquil, Ecuador; melissa_carvajalp@hotmail.com; (D) https://orcid. org/0000-0002-9303-5717

2. Médico; Investigadora Independiente; Guayaquil, Ecuador; arianna1medik@hotmail.com; (D) https://orcid. org/0000-0002-3910-4961

3. Médico; Investigadora Independiente; Guayaquil, Ecuador; maveca2786@hotmail.com; (D) https://orcid. org/0000-0001-9540-7476

4. Médico; Investigadora Independiente; Guayaquil, Ecuador; shirleyvi16co@hotmail.com; (D) https://orcid. org/0000-0003-4164-6732

CORRESPONDENCIA

Melissa Monserrat Carvajal Prado

melissa_carvajalp@hotmail.com

\section{Guayaquil, Ecuador}




\title{
RESUMEN
}

La Insuficiencia renal crónica es una de las complicaciones más notables de la Diabetes Mellitus, cuya propagación afecta cada día más a las personas alrededor del mundo, a medida que se expande la influencia de la diabetes a escala masiva. La comprensión de la conjunción entre estas enfermedades no transmisibles, que ha dado lugar a la Nefropatía diabética, es de vital importancia para afrontar de forma eficiente esta patología, en función de brindar a los pacientes un esquema que permita mitigar su desarrollo acelerado. En el presente trabajo, se realiza una revisión documental donde se abordan elementos fisiopatológicos de la enfermedad, clasificación, estadios, factores de riesgo, patogénesis, tratamiento entre otros aspectos claves que aportan una visión holística de esta patología. Se otorga especial relevancia al glomérulo como unidad donde se desarrollaran las alteraciones principales en el riñón diabético. Finalmente se evidencia la importancia del control glucémico y el control de la presión arterial como variables fundamentales un efectivo manejo de los pacientes con este tipo de afectación.

Palabras clave: Diabetes Mellitus, Insuficiencia Renal Crónica, Glomérulo, Nefropatía diabética.

\begin{abstract}
Chronic renal failure is one of the most notable complications of Diabetes Mellitus, which spread affects people around the world every day, proportionally to the expansion of the diabetes influence on a massive scale. The understanding of the conjunction between these non transmittable diseases that has led to the diabetic nephropathy is of vital importance to face efficiently this pathology, providing patients a scheme that mitigates its accelerated development. In the present work, a documentary review is carried out where physio pathological elements of the disease are addressed, classification, stages, risk factors, pathogenesis, and treatment among other key aspects that allow a holistic view of this pathology. Special importance is given to the glomerulus as a unit where the main alterations are developed in the diabetic kidney. Finally, the importance of glycemic control and blood pressure control is evidenced as fundamental variables for an effective management of patients with this type of disease.
\end{abstract}

Keywords: Diabetes Mellitus, Chronic Renal Failure, Glomerulus, Diabetic Nephropathy.

\section{RESUMO}

A insuficiência renal crônica é uma das complicações mais notáveis do Diabetes Mellitus, que se espalha afeta diariamente pessoas do mundo todo, proporcionalmente à expansão da influência do diabetes em grande escala. $\mathrm{O}$ entendimento da conjunção entre essas doenças não transmissíveis que levaram à nefropatia diabética é de vital importância para o enfrentamento eficiente dessa patologia, fornecendo aos pacientes um esquema que atenua seu desenvolvimento acelerado. No presente trabalho, é realizada uma revisão documental onde são abordados os elementos fisiopatológicos da doença, classificação, estágios, fatores de risco, patogênese e tratamento, entre outros aspectos-chave que permitem uma visão holística dessa patologia. É dada especial importância ao glomérulo como uma unidade em que as principais alterações são desenvolvidas no rim diabético. Por fim, evidencia-se a importância do controle glicêmico e da pressão arterial como variáveis fundamentais para um manejo eficaz de pacientes com esse tipo de doença.

Palavras-chave: Diabetes Mellitus, Insuficiência Renal Crônica, Glomérulo, Nefropatia Diabética. 


\section{Introducción}

Para el año 2016 la Organización Mundial de la Salud (OMS) estimo que 422 millones de personas adultas en el mundo padecían de diabetes, casi triplicando la cantidad de diabéticos registrado en el año 1980, alcanzando niveles de epidemia global. En su Informe Mundial sobre la Diabetes, la OMS, indica además que "en 2012, la diabetes provocó 1,5 millones de muertes. Un nivel de glucosa en la sangre superior al deseable provocó otros 2,2 millones de muertes, al incrementar los riesgos de enfermedades cardiovasculares y de otro tipo" (Organización Mundial de la Salud, 2016)
Particularmente en poblaciones de bajos o medianos ingresos, la cantidad de decesos vinculados a la diabetes es mayor, debido a los altos costos de los exámenes para determinar de qué tipo de diabetes se trata y sus respectivos tratamientos.

En el caso particular de Ecuador, el perfil de la diabetes publicado en 2016 por esta organización, indica que son atribuibles a esta enfermedad un $4 \%$ de las muertes totales, ubicándose entre los países con un grupo de ingresos medianos - altos y observándose una tendencia ligeramente creciente en los últimos 10 años, como puede observarse en la grafica siguiente.

\section{Ecuador}

\section{Mortalidad}

Número de muertes por diabetes

30-69 años

70 años o más

Mortalidad proporcional (\% del total de muertes, todas las edades)*

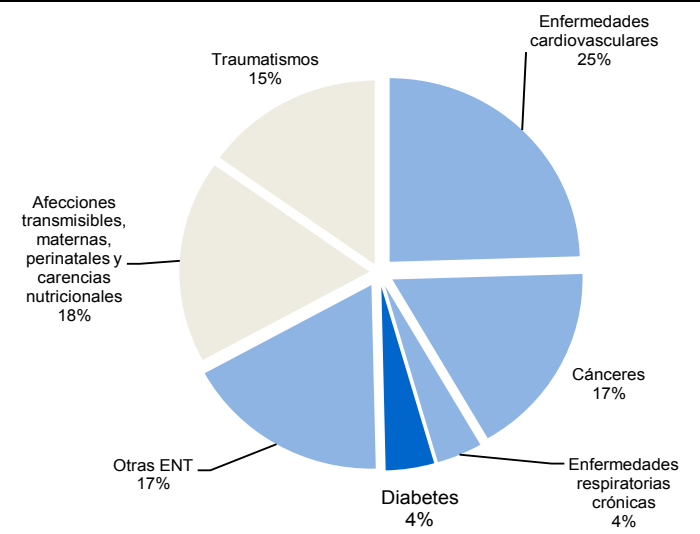

Número de muertes atribuibles a la hiperglucemia

\begin{tabular}{lcc}
$30-69$ años & hombres & mujeres \\
\cline { 2 - 3 } 70 años o más & 1010 & 1000 \\
& 1350 & 2360
\end{tabular}

Tendencias en la diabetes estandarizadas por edades

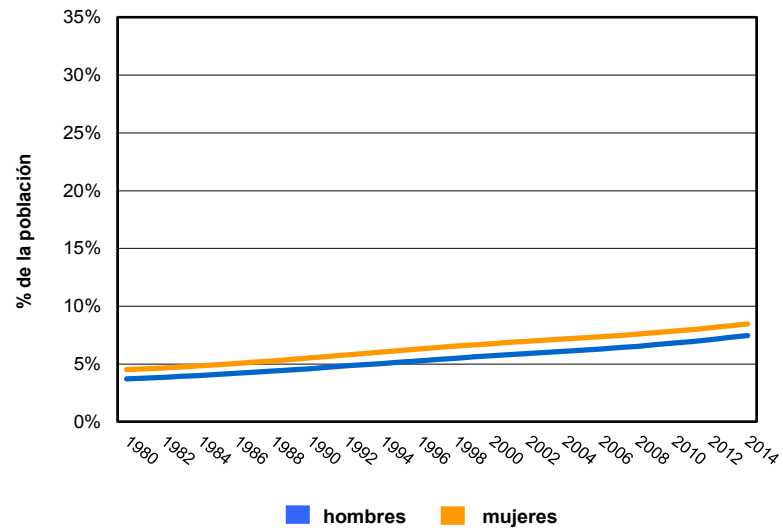

Prevalencia de la diabetes y de los factores de riesgo conexos

\begin{tabular}{|l|c|c|c|}
\hline & hombres & mujeres & \multicolumn{2}{c|}{ total } \\
\hline Diabetes & $6.7 \%$ & $7.9 \%$ & $7.3 \%$ \\
\hline Sobrepeso & $50.1 \%$ & $55.5 \%$ & $52.8 \%$ \\
\hline Obesidad & $13.9 \%$ & $22.2 \%$ & $18.0 \%$ \\
\hline Inactividad física & $18.9 \%$ & $29.9 \%$ & $24.5 \%$ \\
\hline
\end{tabular}

Figura 1. Diabetes mellitus en Ecuador

Fuente: (OMS, 2016) 
De las diversas consecuencias que genera la Diabetes Mellitus(DM), la insuficiencia renal crónica (IRC) es una de las complicaciones más comunes, particularmente en pacientes con diabetes mellitus tipo 2(DM2), así mismo la Organización Panamericana de la Salud, indica que la diabetes y la hipertensión, especialmente en adultos mayores, constituyen factores de riesgo fundamentales para el desarrollo de la enfermedad renal crónica, afectando a uno de cada diez adultos alrededor del mundo, con el agravante de que en muchos casos, estas Enfermedades no Transmisibles (ENT) son detectadas luego de varios años de desarrollo, por lo cual ambas son hoy consideradas problemas de salud pública por parte de los gobiernos mundiales, haciéndose necesario diseñar estrategias para su prevención y tratamiento efectivo.

Al evaluar la correlación entre diabetes mellitus e insuficiencia renal, puede observarse la determinante incidencia de la glucosa en la micro vasculatura glomerular del riñón y por ende en toda su estructura, afectando entre otras cosas, la tasa de filtración glomerular para posteriormente permitir el paso de albúmina, lo cual constituye uno de los principales marcadores de la insuficiencia renal, con graves consecuencias para el paciente.

La estrecha vinculación entre la diabetes mellitus y la insuficiencia renal, obliga a consensuar métodos y parámetros de intervención que permitan aminorar las fatales consecuencias que la IRC puede tener en los pacientes diabéticos.

\section{Metodología}

El propósito del presente trabajo de investigación es realizar una revisión documental en función de la valorar los elementos esenciales y perspectivas acerca de la correlación entre estas dos enfermedades, conjugadas en lo que se conoce hoy como nefropatía diabética, este sentido la metodología asumida es la investigación documental definida por Fidias Arias como: "...un proceso basado en la búsqueda, recuperación, análisis, crítica e interpretación de datos secundarios, es decir, los obtenidos y registrados por otros investigadores en fuentes documentales: impresas, audiovisuales o electrónicas. Como en toda investigación, el propósito de este diseño es el aporte de nuevos conocimientos." (Arias, 2012)

Para enriquecer la búsqueda documental se accedieron a varios centros de investigación, artículos científicos y publicaciones institucionales, los cual permitieron tener una comprensión integral sobre el problema de la insuficiencia renal en pacientes con diabetes mellitus.

Como punto de partida se precisó la revisión documental de algunos conceptos básicos relacionados con el objeto de la investigación, como lo son la diabetes mellitus, la insuficiencia renal crónica, glomérulo y tasa de filtración glomerular.

La Organización Mundial de la Salud (2019) define la diabetes como:

“...una enfermedad crónica que aparece cuando el páncreas no produce insulina suficiente o cuando el organismo no utiliza eficazmente la insulina que produce. El efecto de la diabetes no controlada es la hiperglucemia (aumento del azúcar en la sangre)." (World Health Organization, 2019)

Por otra parte Según Conget, la define de la siguiente forma:

"la diabetes mellitus (DM) es un trastorno metabólico caracterizado por la presencia de hiperglucemia crónica acompañado de un deterioro mayor o menor en el metabolismo de carbohidratos, lípidos y proteínas. El origen y la etiología de la DM pueden variar mucho, pero puede incluir defectos en la secreción o respuesta de insulina o en ambos en algún momento de la enfermedad. Cuando los síntomas característicos de la DM están claramente presentes y los niveles de glucosa en sangre son lo suficiente- 
mente altos, el diagnóstico es generalmente inequívoco." (Conget, 2002)

En cuanto a la insuficiencia renal crónica (IRC), Gómez la define como:

"la pérdida progresiva, permanente e irreversible de la tasa de filtración glomerular a lo largo de un tiempo variable, a veces incluso de años, expresada por una reducción del aclaramiento de creatinina estimado $<60 \mathrm{ml} / \mathrm{min} / 1,73 \mathrm{~m} 2$ (1)." (Gomez, 2007)

Una vez aclaradas las definiciones de ambas patologías, es fundamental atender a la fisiología del glomérulo, el cual constituirá el centro de las alteraciones ocasionadas por la influencia de la glucosa presente en el torrente sanguíneo sobre el riñón, la cual terminará por causar la insuficiencia renal crónica.

De acuerdo con Acosta y Verdague:

"el glomérulo consiste en un ramillete de capilares sostenido por un tejido conectivo (mesangio), recubierto de una doble estructura membrano-epitelial, que se forma a partir de una dilatación e invaginación en forma de copa del extremo ciego del túbulo contorneado proximal (TCP). La capa interna de esta doble estructura se adosa a los capilares y mesangio a modo de un manto, formando parte de la pared capilar; la capa externa, en continuidad con el manto membrana-epitelial por un lado y con la membrana basal y epitelio del TCP por el otro, forma la llamada cápsula de Bowman." (Verdague, 1990)

La siguiente imagen presenta una ilustración referencial descriptiva del glomérulo y sus partes, entre las cuales pueden destacarse, las arteriolas aferentes y eferentes, cápsula de bowman, membrana basal glomerular(MBG), matriz mesangial, células mesangiales, entre otras que sufrirán alteraciones importantes en pacientes diabéticos.

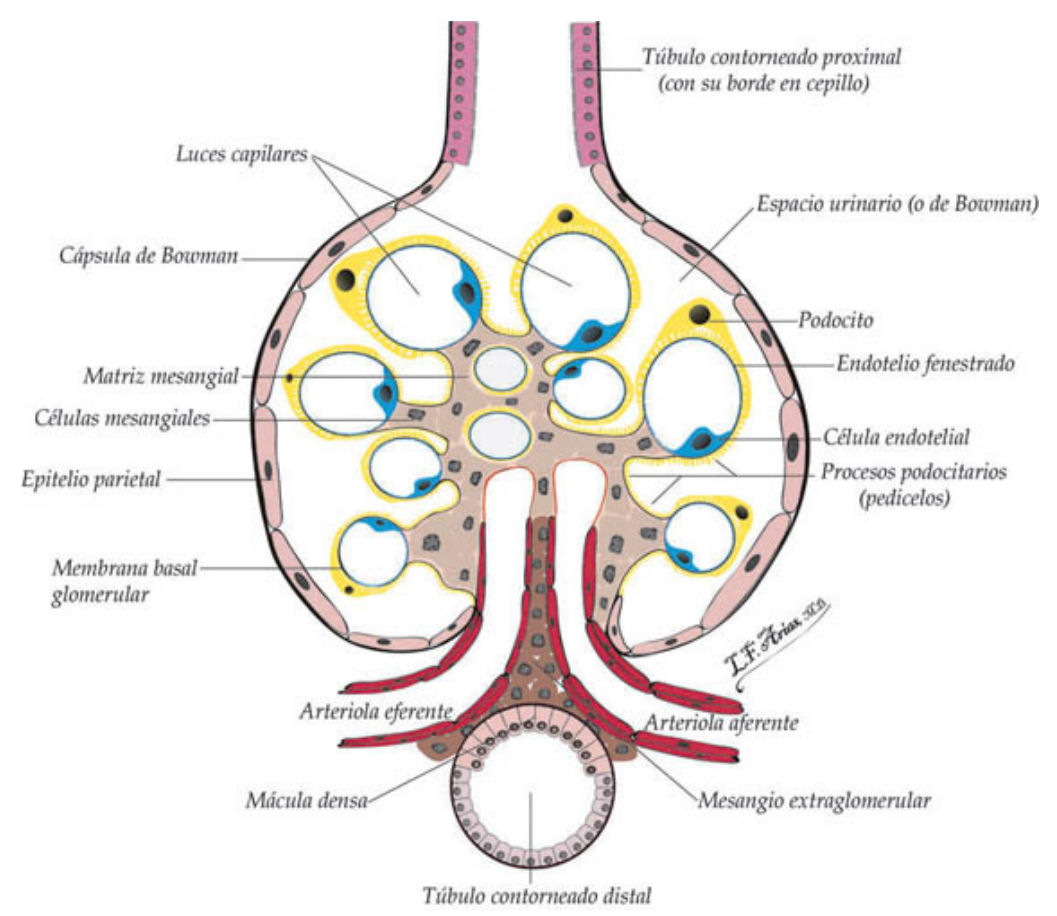

Figura 2. El glomérulo

Fuente: (Bariety J., 2016)

Otro concepto clave a considerar es la Tasa de filtración glomerular (TFG), el cual básicamente es el volumen de fluido filtrado por unidad de tiempo desde los capilares glomerulares renales hacia el interior de la cápsula de Bowman. Se mide en mililitros por minuto ( $\mathrm{ml} / \mathrm{min})$. Este constituye un indicador fundamental para evaluar el funcio- 
namiento del riñón y es también uno de los indicadores - variables a analizar en casos de diabetes mellitus.

La insuficiencia renal crónica causada por la diabetes es también conocida como nefropatía diabética, este síndrome fue descrito por primera vez por el médico británico Clifford Wilson y el médico estadounidense Paul Kimmelstiel en 1936, se define como una afección de la vasculatura renal, ocasionada por la diabetes, a raíz de cambios morfológicos y estructurales causados por la presencia de residuos de glucosa en la estructura del glomérulo. Así lo afirma una investigación publicada en la Revista MedWave, realizada por varios autores, quienes definen la nefropatía diabética como:

"una complicación causada por la diabetes a nivel del micro vasculatura renal. Los individuos con diabetes presentan una mayor tasa de filtración glomerular o hiperfiltración, mediada por la mayor relajación de las arteriolas aferentes en comparación a las eferentes. A su vez, esto conduce a un aumento del flujo sanguíneo a través del capilar glomerular, elevando la presión. Cuando estas condiciones se mantienen en el tiempo, producen tanto una hipertrofia glomerular como un aumento de la superficie del capilar glomerular. Ello causa alteraciones hemodinámicas que contribuyen al desarrollo y/o progresión de esta enfermedad" (Carlos Eduardo Meza Letelier, 2017)

Otros autores como Torres y Castillo (2002) hacen referencia a la evolución del término indicando que inicialmente este se usaba para identificar cualquier lesión del riñón diabético, sin embargo actualmente se refiere a lesiones renales originadas por afección en los vasos renales específicamente, provocando alteraciones estructurales del riñón a nivel glomerular.

En términos generales, los marcadores que pueden observarse en un paciente que padece nefropatía diabética son los siguientes:
- Albuminuria mayor a 300mg/dl confirmada en 2 ocasiones en un lapso temporal de 3 a 6 meses, lo cual se identifica mediante examen sanguíneo.

- Alteración de la tasa de filtración glomerular hasta llegar a valores menores a $120 \mathrm{mg} / \mathrm{dl}$, este se identifica igualmente mediante examen sanguíneo.

- Hipertensión arterial sistémica, esto es, mayor o igual a 130/80 mmHg.

Para llegar a manifestarse estos indicadores, tienen lugar una serie de procesos que inician por la presencia excesiva de glucosa en el torrente sanguíneo, la cual tiende a unirse a moléculas que se encuentran en la sangre, especialmente proteínicas, que al circular por el riñón alteran la estructura completa del glomérulo renal como se describe a continuación. El proceso de adhesión de glucosa a las proteínas se conoce como glicosilación no enzimática de proteínas plasmáticas, en el caso del riñón y sus glomérulos, este proceso afecta particularmente aquellas proteínas ubicadas en el endotelio de la arteriola eferente, alterando drásticamente su estructura, debido a su endurecimiento y engrosamiento que consecuentemente ocasiona un aumento de presión en dicha arteria y la presión hidrostática presente en los capilares glomerulares, limitando el cumplimiento de su función natural y permitiendo en primer momento una mayor filtración del flujo sanguíneo, es decir, alterando la tasa de filtración glomerular mediante híper filtración. Los principales cambios fisiopatológicos en la nefropatía diabética incluyen:

- El engrosamiento de la membrana basal glomerular (GBM),

- La expansión mesangial.

- La esclerosis nodular - cambio de Kimmelstiel Wilson.

- La esclerosis glomerular difusa.

- La fibrosis intersticial tubular y. 
- La arteriosclerosis e hialinósis de los vasos sanguíneos renales.

Cobra especial importancia la barrera de filtración glomerular ya que esta funciona como un tamiz biológico complejo. A diferencia de otros capilares en el cuerpo, los capilares glomerulares son altamente permeables al agua y relativamente impermeables a las moléculas grandes. Tal permeabilidad es posible debido a la estructura única de tres capas de la membrana de filtración glomerular que consiste en una capa endotelial, membrana basal glomerular y células epiteliales viscerales glomerulares. En los glomérulos de pacientes con DM, se dan cambios patológicos de larga duración antes de la aparición de microalbuminuria.

Las afecciones iniciales causan una hipertrofia de la arteriola eferente o arterioesclerosis hialina e hiperfiltración, las cuales a su vez tienen a su vez un impacto importante en la membrana basal glomerular. De igual forma, se ve híper estimulado el sistema renina-angiotensina-aldosterona, el cual consiste en una secuencia de reacciones

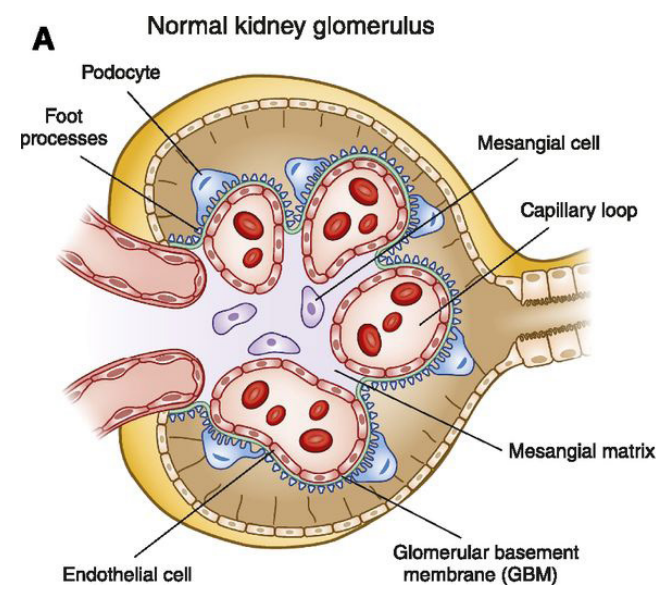

diseñadas para ayudar a regular la presión arterial, este impacto influye e incrementa la presión arterial, derivando una híper tensión arterial sistémica que contribuye a una presión aun mayor en la arteriola eferente. Lo complejo de este cuadro es que las afecciones iniciales tienen en muchos casos un carácter asintomático, sin embargo van a desembocar en un daño estructural de la membrana glomerular, lo cual a su vez dará paso a la aparición de la micro albuminuria, seguida de una disminución de tasa de filtración glomerular, generando finalmente macro albuminuria y con ello insuficiencia renal crónica. La gravedad del daño glomerular es proporcional al valor de la Tasa de Filtración Glomerular, la duración de la Diabetes Mellitus y la regulación de la glucosa en sangre.

En la siguiente imagen puede observarse una ilustración referencial del glomérulo renal normal y el glomérulo renal diabético, en el que puede observarse un engrosamiento de la membrana basal glomerular, la proliferación de células mesangiales y contracción de las células endoteliales.

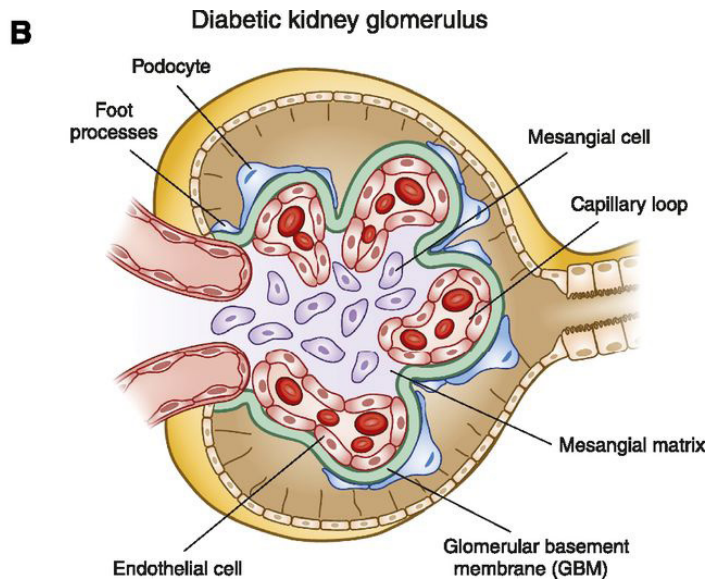

Figura 3. Morfología renal normal y cambios estructurales en diabetes mellitus. La enfermedad renal diabética induce cambios estructurales, que incluyen el engrosamiento de la membrana basal glomerular, la fusión de los procesos del pie, la pérdida de podocitos con el desprendimiento de la membrana basal glomerular y la expansión de la matriz mesangial

Fuente: (Radica Z. Alicic, 2017) 
Dado que los cambios histológicos en ambos tipos de DM se superponen en gran medida, se ha establecido mundialmente una clasificación patológica de la nefropatía diabética, donde la nefropatía diabética se divide histológicamente en cuatro etapas de daño glomerular descritas en la siguiente tabla.

Tabla 1. Clasificación patológica internacional de los cambios glomerulares en el riñón diabético

\begin{tabular}{|c|l|l|}
\hline Clase & \multicolumn{1}{|c|}{ Descripción } & \multicolumn{1}{|c|}{ Criterios de inclusión } \\
\hline 1 & $\begin{array}{l}\text { Microscopía óptica leve o } \\
\text { inespecífica. Cambios y y } \\
\text { microscopía electrónica } \\
\text { engrosamiento MBG } \\
\text { probado }\end{array}$ & $\begin{array}{l}\text { Membrana Glomerular de 395 nm mujeres } \\
\text { y.430 nm 9 años de edad y mayores; la } \\
\text { biopsia no cumple ninguno de los criterios } \\
\text { mencionados a continuación para las } \\
\text { clases 2-4 }\end{array}$ \\
\hline 2 a & $\begin{array}{l}\text { Expansión mesangial } \\
\text { leve }\end{array}$ & $\begin{array}{l}\text { Expansión mesangial leve en .25\% del } \\
\text { mesangio observado; la biopsia no cumple } \\
\text { criterios para la clase 3 o 4 }\end{array}$ \\
\hline $2 \mathrm{~b}$ & $\begin{array}{l}\text { Expansión mesangial } \\
\text { severa }\end{array}$ & $\begin{array}{l}\text { Expansión mesangial severa en .25\% del } \\
\text { mesangio observado; la biopsia no cumple } \\
\text { criterios para la clase 3 o 4 }\end{array}$ \\
\hline 3 & $\begin{array}{l}\text { Esclerósis nodular } \\
\text { (Lesión Kimmelstiel } \\
\text { Wilson) }\end{array}$ & $\begin{array}{l}\text { Al menos una lesión convincente } \\
\text { Kimmelstiel - Wilson; la biopsia no cumple } \\
\text { con los criterios para la clase 4 }\end{array}$ \\
\hline 4 & $\begin{array}{l}\text { Glomeruloesclerosis } \\
\text { diabética avanzada }\end{array}$ & $\begin{array}{l}\text { Esclerosis glomerular global en el 50\% de } \\
\text { los glomérulos; lesiones de las clases 1-3 }\end{array}$ \\
\hline
\end{tabular}

Fuente: (Radica Z. Alicic, 2017)

Una mirada detallada a la progresión de la modificación estructural del glomérulo a raíz de la diabetes mellitus, es aportada por investigadores de la Sociedad Americana de Nefrología, como puedo observarse en las siguientes imágenes, que van desde la figura del glomérulo normal(A) hasta el glomérulo obsoleto $(F)$, en un proceso caracterizado por la expansión mensagial, entre otros indicadores.

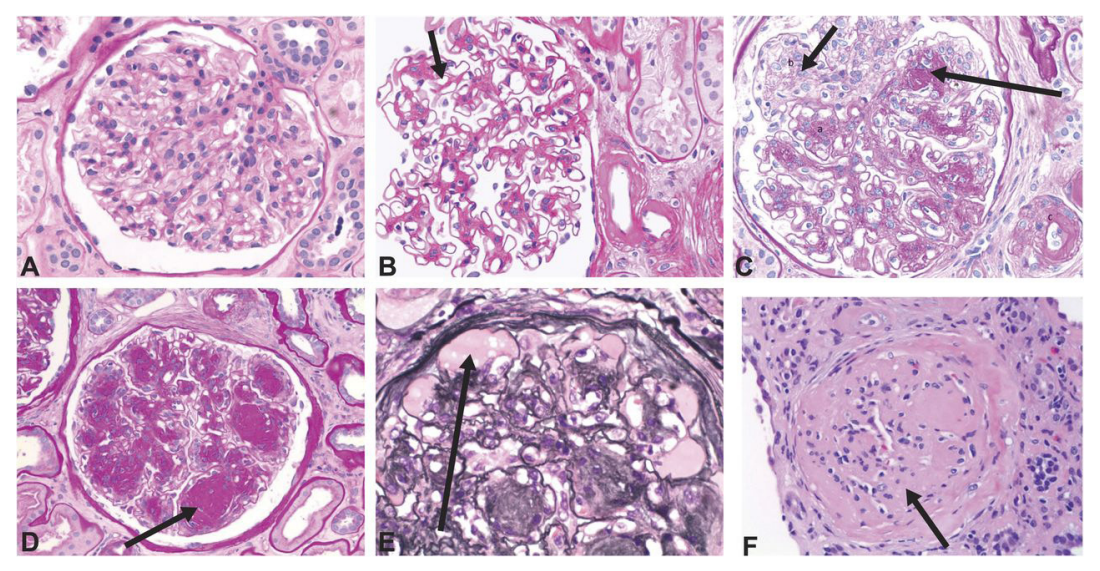

Figura 4. Glomerulopatía diabética

Fuente: (Radica Z. Alicic, 2017) 
Aquí hay una superposición significativa entre los cambios descritos en pacientes en diferentes etapas de albuminuria, independientemente de su tipo de DM. Todos los patrones histológicos tienen un pronóstico idéntico. Sin embargo, el hecho de que la expansión del mesangio y la esclerosis glomerular no ocurran simultáneamente indica su diferente patogénesis dentro de la nefropatía diabética. Bajo microscopía óptica, la reducción en el número de podocitos se nota fácilmente en pacientes con DM tipo 1 y tipo 2.
Más allá de las alteraciones de carácter histológico, otras clasificaciones se orientan hacia un enfoque evolutivo de la patología, basado en el estimado de filtración glomerular, albuminuria y presión arterial, en ese sentido, la Sociedad Latinoamericana de Nefrología e Hipertensión considera comúnmente los siguientes estadios como parte de la secuencia evolutiva de la nefropatía diabética:

Tabla 2. . Estadios de la nefropatía diabética

\begin{tabular}{|c|c|c|c|c|}
\hline Estadio & Características & $\begin{array}{l}\text { Estimado de } \\
\text { Filtrado Glomerular }\end{array}$ & Albuminuria & Presión Arterial \\
\hline $\begin{array}{l}\text { Estadio } 1 \\
\text { Presente al momento } \\
\text { del diagnóstico } \\
\text { de DM }\end{array}$ & $\begin{array}{l}\text { Hiperfiltración } \\
\text { glomerular }\end{array}$ & $\begin{array}{l}\text { Incrementada en DM } \\
1 \text { y } 2\end{array}$ & $\begin{array}{l}\text { Puede estar presente } \\
\text { en forma episódica } \\
\text { y reversible con control } \\
\text { glucémico }\end{array}$ & $\begin{array}{l}\text { DM1: normal } \\
\text { DM2: normal o } \\
\text { incrementada }\end{array}$ \\
\hline $\begin{array}{l}\text { Estadio } 2 \\
\text { Primeros } 5 \text { años }\end{array}$ & $\begin{array}{l}\text { Engrosamiento de } \\
\text { la membrana basal } \\
\text { y expansión del } \\
\text { mesangio }\end{array}$ & Normal & $\begin{array}{l}\text { Puede estar presente } \\
\text { en forma episódica } \\
\text { y reversible con control } \\
\text { glucémico }\end{array}$ & $\begin{array}{l}\text { DM1: normal } \\
\text { DM2: normal o } \\
\text { incrementada }\end{array}$ \\
\hline $\begin{array}{l}\text { Estadio } 3 \\
6 \text { a } 15 \text { años }\end{array}$ & Microalbuminuria & $\begin{array}{l}\text { Normal o disminuido en } \\
\text { relación a su basal }\end{array}$ & 30 a 300 mg/día & $\begin{array}{l}\text { DM1: incrementada } \\
\text { DM2: normal o } \\
\text { incrementada }\end{array}$ \\
\hline $\begin{array}{l}\text { Estadio } 4 \\
15 \text { a } 25 \text { años }\end{array}$ & Macroalbuminuria & $\begin{array}{l}\text { Normal o disminuido y } \\
\text { en descenso progresivo }\end{array}$ & > 300 mg/día & Hipertensión \\
\hline $\begin{array}{l}\text { Estadio } 5 \\
25 \text { a } 30 \text { años }\end{array}$ & $\begin{array}{l}\text { Insuficiencia renal } \\
\text { terminal }\end{array}$ & 0 a $10 \mathrm{ml} / \mathrm{min}$ & Disminuyendo & Hipertensión \\
\hline
\end{tabular}

Fuente: (Rosas Guzmán, y otros, 2016)

En cuanto a la patogénesis de la nefropatía diabética, puede decirse que es resultado de la interacción de factores hemodinámicos y metabólicos, entre los cuales destacan:

- Híper filtración glomerular: cambios tempranos en el aumento de la presión intraglomerular e hiperfiltración fueron descritas por Stadler y Schmidt como parte inicial del desarrollo de la nefropatía diabética en 1959. Posteriormente en la década de 1970, Mogensen enfatizó que hasta un $40 \%$ de los casos de DM recientemente encontrados habían aumentado la filtración glomerular. Aunque el mecanismo de desarrollo de la hiperfiltración no se comprende completamente, se ha encontrado que varios factores juegan un papel en su desarrollo. (Stadler G, 1959)

- Control pobre de factores metabólicos (productos finales de glicación, hiperglucemia, proteína quinasa C): Parte del exceso de glucosa en la hiperglucemia crónica se une a aminoácidos libres de proteínas circulantes o tisulares. Este proceso no enzimático produce productos reversibles de glicación temprana, y más tarde, productos finales de glicación avanzada (AGE) irreversibles, que 
se acumulan en los tejidos y contribuyen al desarrollo de complicaciones microvasculares de la DM. La evidencia de los estudios in vitro muestra que la hiperglucemia tiene un efecto directo sobre la proliferación celular mesangial, la expansión de la matriz y la glicosilación de proteínas glomerulares. La activación de la proteína quinasa C (PKC) es uno de los principales mediadores de la lesión tisular inducida por hiperglucemia. La activación de PCK conduce a una mayor permeabilidad vascular, una mayor síntesis de componentes de la matriz extracelular y una mayor producción de especies reactivas de oxígeno (ROS), que son mediadores importantes de la lesión renal.

- Hormonas: El papel de las hormonas se demostró en un estudio experimental que la infusión de análogos de somatostatina (octreotida) condujo en parte a la disminución de la híper filtración y el tamaño del riñón.

- Sorbitol: La enzima aldosa reductasa convierte la glucosa intracelular en sorbitol, que permanece en la célula. Si bien la investigación en pacientes con DM tipo 1 e hiperfiltración conocida ha demostrado que la infusión de inhibidor de la aldosa reductasa (tolrestat) disminuye la TFG a valores normales, un posible uso terapéutico de este agente debería confirmarse en más estudios. (Passariello N, 1993)

- Mayor reabsorción de sodio y retroalimentación tubuloglomerular: El aumento de la reabsorción de sodio tubular renal debido al aumento del cotransporte de sodio y glucosa conduce al aumento del volumen de líquido extracelular, que luego aumenta la TFG. (Vallon V, 2003)

- Expresión de heparanasa: La regulación de la expresión de heparanasa juega un papel importante en la patogénesis de la nefropatía diabética. La reducción del sulfato de heparina en la superficie de las células endoteliales cambia la carga negativa de glucocalix y, en consecuencia, aumenta la permeabilidad de la albúmina de la membrana de filtración glomerular.

- Prorenina: El aumento de la prorenina sérica desempeña un papel en el desarrollo de la nefropatía diabética en niños y adolescentes. La prorenina se une a un receptor de tejido específico, lo que lleva a la activación de la vía de señal de las proteínas quinasas activadoras de mitógeno (MAPK), que potencian el desarrollo de daño renal.

- Citoquinas y factores de crecimiento: La hiperglucemia estimula una mayor expresión de diferentes factores de crecimiento y la activación de las citocinas, lo que en general contribuye a un mayor daño renal. (Navarro-Gonzalez JF, 2008)

\section{Factores de Riesgo}

Es importante destacar que existen una serie de Factores de riesgo para el desarrollo de la nefropatía diabética, entre los cuales se consideran factores demográficos, hereditarios, condiciones sistémicas, lesiones renales previas y dietéticas. Estos se clasifican entre factores de susceptibilidad, como edad, genero, raza, etnia; factores de iniciación, como la nefropatía genética, la hiperglucemia, la obesidad, y factores de progresión como la alta ingesta de proteínas, siendo los de mayor impacto la hiperglucemia y la hipertensión. 
Tabla 3. Factores de riesgo

\begin{tabular}{|c|c|c|c|}
\hline Factor de riesgo & Susceptibilidad & Iniciación & Progresión \\
\hline \multicolumn{4}{|l|}{ Demográficos } \\
\hline Edad avanzada & + & & \\
\hline Genero(masculino) & + & & \\
\hline $\begin{array}{c}\text { Raza/etnia(negro, } \\
\text { indio americano, } \\
\text { asiáticos) }\end{array}$ & + & & + \\
\hline \multicolumn{4}{|l|}{ Hereditarios } \\
\hline $\begin{array}{l}\text { Historia familiar } \\
\text { con nefropatía } \\
\text { diabética }\end{array}$ & + & & \\
\hline $\begin{array}{l}\text { Nefropatía } \\
\text { genética }\end{array}$ & & + & \\
\hline \multicolumn{4}{|l|}{$\begin{array}{l}\text { Condiciones } \\
\text { sistémicas }\end{array}$} \\
\hline Híper glicemia & + & + & + \\
\hline Obesidad & + & + & + \\
\hline Hipertensión & + & & + \\
\hline \multicolumn{4}{|l|}{ Lesiones en riñón } \\
\hline $\begin{array}{c}\text { Insuficiencia renal } \\
\text { aguda }\end{array}$ & & + & + \\
\hline Toxinas & & + & + \\
\hline Fumador & + & & + \\
\hline \multicolumn{4}{|l|}{$\begin{array}{l}\text { Factores } \\
\text { dietéticos }\end{array}$} \\
\hline $\begin{array}{c}\text { Alta ingesta de } \\
\text { proteínas }\end{array}$ & + & & + \\
\hline
\end{tabular}

Fuente: (Radica Z. Alicic, 2017)

\section{Control glucémico estricto}

El efecto del control glucémico estricto depende de la etapa de DM en la que se inició y la consiguiente normalización del metabolismo de la glucosa. La terapia intensificada con insulina tiene los siguientes efectos en el riñón:

1. Reduce en parte la hipertrofia e hiperfiltración glomerular (en ayunas y después de una comida rica en proteínas), los cuales son factores de riesgo importantes para el daño glomerular permanente.

2. b. Pospone el desarrollo de albuminu- ria. La terapia intensificada con insulina que mantiene los valores de glucosa dentro de los rangos normales disminuye el desarrollo o el progreso de la nefropatía diabética.

3. Estabiliza o disminuye la eliminación de proteínas en pacientes con proteinuria pronunciada.

4. Reduce el progreso de la enfermedad renal en caso de proteinuria ya desarrollada confirmada por método semicuantitativo.

5. Reduce el número de células mesangia- 
les y la matriz mesangial.

6. En algunos pacientes, el grosor de las membranas basales glomerulares y tubulares y el número de células mesangiales se normalizan y los nódulos glomerulares desaparecen.

7. El progreso de la atrofia tubular se ralentiza.

\section{Control estricto de la presión arterial}

El control estricto de la presión arterial es importante en la prevención del progreso de la nefropatía diabética y otras complicaciones en pacientes con DM. El rango óptimo más bajo de presión arterial sistólica no está claramente definido. Según el estudio UKPDS, una reducción de la presión arterial sistólica de $10 \mathrm{~mm} \mathrm{Hg}$ disminuye el riesgo de desarrollar complicaciones diabéticas en un 12\%; el riesgo es el más bajo cuando los valores de presión arterial sistólica son inferiores a $120 \mathrm{~mm} \mathrm{Hg}$.

El ensayo de nefropatía diabética de Irbesartan demostró que la disminución de la presión arterial sistólica al valor límite inferior de $120 \mathrm{~mm} \mathrm{Hg}$ reduce el riesgo de mortalidad cardiovascular e insuficiencia cardíaca (pero no de infarto de miocardio). De acuerdo con las Directrices actuales sobre el tratamiento de la hipertensión arterial (Sociedad Europea de Hipertensión, 2007) la presión arterial objetivo en pacientes con DM debe ser menor a 130/80 mm Hg. La terapia antihipertensiva puede iniciarse incluso cuando los valores de presión arterial están en el rango normal superior.

\section{Dislipidemia}

La dislipidemia ocurre en todos los pacientes con DM, y su aparición aumenta con el desarrollo de la nefropatía diabética. La reducción agresiva de lípidos plasmáticos es una intervención terapéutica importante, porque los pacientes con DM tienen un mayor riesgo de enfermedad coronaria. Además, la dislipidemia contribuye al desarrollo de la nefropatía diabética. El tratamiento de la dislipidemia con estatinas ralentiza la progresión de la nefropatía diabética.

\section{Conclusiones}

En los últimos años, la ciencia ha alcanzado un importante progreso en la comprensión de los factores de riesgo y el mecanismo del desarrollo de la nefropatía diabética, así como en las posibilidades de tratamiento destinadas a prevenir la progresión de la enfermedad. La detección temprana de esta complicación crónica de la DM junto con el tratamiento de los principales factores de riesgo (hiperglucemia, hipertensión y dislipidemia) y el uso de medicamentos renoprotectores (IECA y BRA) pueden disminuir la progresión de esta enfermedad renal.

El tratamiento del aumento de la presión arterial es una prioridad para mitigar los riesgos de la nefropatía diabética, esto en conjunto con otras medidas pueden conducir a una disminución de la mortalidad general y cardiovascular en pacientes con DM.

La albuminuria constituye el biomarcador convencional para la detección y predicción de la nefropatía diabética y el consecuente riesgo cardiovascular en pacientes con DM1 y DM2. Existen también biomarcadores novedosos que tienen mayor sensibilidad y especificidad para la detección temprana de DN, así como para una predicción más precisa de su progresión a una enfermedad renal terminal.

La nefropatía diabética es consecuencia de distintas variables, como lo son las variantes genéticas, efectos epigenéticos e interacciones relacionadas con el medio ambiente. Los estudios que aclaran los mecanismos subyacentes detrás de esta patología están aun en desarrollo investigativo. Sin embargo, docenas de nuevas tecnologías de análisis y medidas farmacológicas están en auge. Con el progreso de mas investigaciones especializadas, sobre los mecanismos de inicio y progresión de esta enfermedad, las posibles respuestas serán finalmente 
1 exploradas y arrojarán mayores luces sobre su patogénesis, facilitarán la prevención, permitirán un diagnóstico oportuno y la intervención personalizada para reducir su incidencia y minimizar su progresión, para aliviando de esta forma la carga que representa para la salud pública.

\section{Bibliografía}

Arias, F. G. (2012). El Proyecto de Investigación. Caracas, Venezuela: Episteme.

Bariety J., M. C. (2016). Histología Normal. Antioquia, Colombia: Nefropatología. Obtenido de https:// www.kidneypathology.com/Histologia_glomerulo. $\mathrm{html}$

Carlos Eduardo Meza Letelier, C. A. (2017). Fisiopatologia de la nefropatóa diabética. MedWave.

Conget, D. I. (2002). Diagnóstico, clasificación y patogenia. Revista Española de Cardiología, 528.

Gomez, A. J. (2007). Tratado de Geriatria para residentes. España: SEGG.

Navarro-Gonzalez JF, M.-F. C. (2008). El papel de las citocinas inflamatorias en la nefropatia diabetica. Diario Clinico de la Asociacion Americana de $\mathrm{Ne}$ frología, 433-442.

OMS. (2016). Número de muertes por diabetes. Ginebra, Suiza: Organización Mundial de la Salud.

Organización Mundial de la Salud. (2016). Informe Mundial sobre la Diabetes. ONU.

Passariello N, S. J. (1993). Efecto del inhibidor de la aldosa reductasa (tolrestat) sobre la tasa de ex- creción de albúmina urinaria y la tasa de filtración glomerular en sujetos con DMID con nefropatía.

Radica Z. Alicic, M. T. (2017). Diabetic Kidney Disease: Challenges, Progress, and Possibilities. Clinical Journal of American Society of Nefrology, 12(12), 2032-2045. doi:10.2215/CJN.11491116

Rosas Guzmán, J., Gómez Pérez, F., Barceló, A., Correa-Rotter, R., Valdivia Lópe, J., \& Calvagno, M. (2016). Prevención, diagnóstico y tratamiento temprano de la nefropatía diabética. Recomendaciones de la Asociación Latinoamericana de Diabetes(ALAD). Avalado por la Sociedad Latinoamericana de Nefrologia e hipertensión. Consensos ALAD, 32(1), 1-8. Recuperado el 2019, de http://alad-americalatina.org/wp-content/ uploads/2016/10/PREVENCION-DE-NEFROPATIA. pdf

Sociedad Europea de Hipertensión. (2007). Directrices para el tratamiento de la hipertensión arterial. 25.

Stadler G, S. R. (1959). Severe funcional disorders of glomerular capillaries and renal hemodynamics in treated diabetes mellitus during childhood. Ann. pediatr.

Vallon V, B. R. (2003). Hiperfiltración glomerular y la paradoja de la sal en raramente diabetes mellitus tipo 1: una visión tubulocéntrica. Clinical Journal of the American Society of Nefrology.

Verdague, D. N. (1990). Hístologia Glomerular Normal. Revista Médica de Uruguay Volumen $6 \mathrm{~N}^{\circ} 3$, 157.

World Health Organization. (2019). World Health Organization. Recuperado el 2019, de https://www. who.int/topics/diabetes_mellitus/es/

\section{CITAR ESTE ARTICULO:}

Carvajal Prado, M., Motoche Granados, K., Vera Carrera, M., \& Vite Correa, S. (2020). Insuficiencia renal crónica en pacientes con diabetes mellitus. RECIMAUC, 114-126. doi:10.26820/reciamuc/4.(1).enero.2020.114-126

(c) $)$ (1) $\circledast$ ()

RECONOCIMIENTO-NOCOMERCIAL-COMPARTIRIGUAL CC BY-NC-SA

ESTA LICENCIA PERMITE A OTROS ENTREMEZCLAR, AJUSTAR Y CONSTRUIR A PARTIR DE SU OBRA CON FINES NO COMERCIALES, SIEMPRE Y CUANDO LE RECONOZCAN LA AUTORÍA Y SUS NUEVAS CREACIONES ESTÉN BAJO UNA LICENCIA CON LOS MISMOS TÉRMINOS. 